TAIWANESE JOURNAL OF MATHEMATICS

Vol. 19, No. 1, pp. 51-63, February 2015

DOI: $10.11650 /$ tjm.19.2015.3357

This paper is available online at http://journal.taiwanmathsoc.org.tw

\title{
SOLVING NONLINEAR COMPLEMENTARITY PROBLEM BY A SMOOTHING HOMOTOPY METHOD
}

\author{
Xiaona Fan*, Tingting $\mathrm{Xu}$ and Furong Gao
}

\begin{abstract}
In this paper, a smoothing homotopy method for solving the nonlinear complementarity problem is considered. The homotopy equation is constructed based on Chen-Harker-Kanzow-Smale smooth function. Under certain mild nonmonotone condition, the global convergence result is obtained. Furthermore, the initial point can be chosen almost everywhere in $R^{n}$ but not just in $R_{+}^{n}$. The numerical experimental results show that the method is effective.
\end{abstract}

\section{INTRODUCTION}

Consider the nonlinear complementarity problem (NCP for abbreviation): Find a vector $x \in R^{n}$ such that

$$
x \geq 0, F(x) \geq 0, x^{T} F(x)=0,
$$

where $F: R^{n} \rightarrow R^{n}$ will be assumed to be continuously differentiable in this paper.

The NCP has attracted much attention due to its various applications in optimization and control problems, game theory, mechanics, operations research and engineering sciences etc.. We refer the reader to $[4,5,6,7]$ for review. For most existing algorithms in the literature, the convergence can be ensured by assuming the mapping $F$ with certain monotonicity, e.g., [3, 8, 9, 11, 14]. In [16], Xu et al. considered to solve the NCP by a homotopy method. They rewrote the NCP as the following system

$$
(x, y) \geq 0, F(x, y)=\left(\begin{array}{c}
X y \\
y-f(x)
\end{array}\right)=0,
$$

Received June 1, 2013, accepted April 24, 2014.

Communicated by Juan Enrique Martinez-Legaz.

2010 Mathematics Subject Classification: 90C33, 90C30, 65C20, 65L05.

Key words and phrases: Nonlinear complementarity problem, Homotopy method, Smoothing method, Global convergence.

This work is supported by the Natural Sciences Foundation for Colleges and Universities in Jiangsu Province of China under Grant No. 11KJB110008, and the Natural Science Foundation of China under Grant No. 11126241, 11201240, 11201241, and Jiangsu Government Scholarship for Overseas Studies.

*Corresponding author. 
where $X=\operatorname{diag}(x)$ is a diagonal matrix formed by the components of $x$. The homotopy equation is constructed as follows:

$$
H\left(w, w^{(0)}, \mu\right)=\left(\begin{array}{c}
X y-\mu X^{(0)} y^{(0)} \\
y-(1-\mu) f(x)-\mu y^{(0)}
\end{array}\right)
$$

where $w^{(0)}=\left(x^{(0)}, y^{(0)}\right) \in R_{++}^{n} \times R_{++}^{n}, w=(x, y) \in R^{n} \times R^{n}, x=\left(x_{1}, \cdots, x_{n}\right)^{T}$, $y=\left(y_{1}, \cdots, y_{n}\right)^{T}, X^{(0)}=\operatorname{diag}\left(x^{(0)}\right)$. They proved that for almost all starting point $x^{(0)} \in R_{+}^{n}$, a finite homotopy pathway formed by the homotopy equation (3) existed and converged to a solution of NCP under a nonmonotone and easily verified convergence condition. For comprehension, we make a slight modification in statement and the condition is described as follows.

Assumption 1. For any $x \subseteq R_{+}^{n}, \lim _{\|x\| \rightarrow \infty} F(x)>0$.

In [16], $\mathrm{Xu}$ et al. have checked that Assumption 1 is not stronger than that of function is monotone or $P_{0}$ and it is very easy to verify.

In this paper, in order to reduce the dimension of variables and enlarge the scope of the initial point, we consider a smoothing homotopy method to solve $\mathrm{NCP}(1)$. We reformulate the NCP as a system of nonsmooth equations via NCP-function[5] $\varphi$ : $R^{2} \rightarrow R:$

$$
\varphi(a, b)=0 \Longleftrightarrow a, b \geq 0, a b=0 .
$$

There are many NCP functions in form, among them we take $\varphi(a, b)=\min (a, b)$, where $\min \{a, b\}$ is the componentwise minimization operator. However, min function is not differentiable, so we reformulate $\min (a, b)=\frac{1}{2}\left(a+b-\sqrt{(a-b)^{2}}\right)$ whose smooth form is given by B. Chen, P. T. Harker, C. Kanzow and S. Smale[2]

$$
\varphi_{C H K S}(a, b, \mu)=\frac{a+b-\sqrt{(a-b)^{2}+4 \mu^{2}}}{2},(a, b)^{T} \in R^{2}, \mu>0 .
$$

After some simple analysis, we obtain several nice properties on this smooth function:

Proposition 1. (i) $\varphi_{C H K S}(a, b, \mu)$ converges to $\min (a, b)$ uniformly as $\mu \rightarrow 0$, and $\left|\varphi_{C H K S}(a, b, \mu)-\min (a, b)\right| \leq \mu$.

(ii) $\varphi_{C H K S}(a, b, 0)=\min (a, b)$.

(iii) For fixed $\mu>0, \varphi_{C H K S}(a, b, \mu)$ is smooth.

Proof. Since 


$$
\begin{aligned}
0 & \geq \varphi_{C H K S}(a, b, \mu)-\min (a, b) \\
& =\frac{a+b-\sqrt{(a-b)^{2}+4 \mu^{2}}}{2}-\frac{a+b-\sqrt{(a-b)^{2}}}{2} \\
& =\frac{1}{2}\left(\sqrt{(a-b)^{2}}-\sqrt{(a-b)^{2}+4 \mu^{2}}\right) \\
& =\frac{-4 \mu^{2}}{2\left(\sqrt{(a-b)^{2}}+\sqrt{(a-b)^{2}+4 \mu^{2}}\right)} \\
& \geq \frac{-4 \mu^{2}}{4 \mu}=-\mu,
\end{aligned}
$$

thus (i) holds. Both (ii) and (iii) are easy to follow.

Remark 1. The above derivation shows $\varphi_{C H K S}(a, b, \mu) \rightarrow \min (a, b)$, when $\mu \rightarrow 0$ and there exists $\alpha(a, b, \mu)$ such that $|\alpha(a, b, \mu)| \leq 1$ and $\varphi_{C H K S}(a, b, \mu)=\min (a, b)-$ $\mu \alpha(a, b, \mu)$.

By the smooth function (4), we can obtain the smooth proximal equation of the NCP:

$$
\Theta(x, \mu)=\left(\begin{array}{c}
\varphi_{C H K S}\left(x_{1}, F_{1}(x), \mu\right) \\
\vdots \\
\varphi_{C H K S}\left(x_{n}, F_{n}(x), \mu\right)
\end{array}\right)=0, \mu>0 .
$$

Based on (5), a homotopy equation can be constructed. The following main content is to prove, under certain suitable condition, when $\mu \downarrow 0$, for almost all $x^{(0)} \in R^{n}$, the homotopy equation converges to the point $\left(x^{*}, 0\right)$. Correspondingly, $x^{*}$ solves equation (5), thus $x^{*}$ is a solution of the NCP.

The remainder of this paper is organized as follows. In Section 2, the main results of the paper are given. A smoothing homotopy equation is constructed, then under Assumption 1, the existence and convergence of a smooth path from almost all given initial point in $R^{n}$ but not $R_{+}^{n}$ in [16] to a solution of the NCP are proved. The numerical results show this smoothing homotopy method for solving the NCP is more effective than that of [16] in Section 3.

\section{Homotopy Method For NCP AND Its CONVERGENCE}

Above all, we introduce several lemmas which are from differential topology and it will be used in the proposed homotopy method. Denote $\psi^{-1}(u)=\{z \mid \psi(z)=u\}$.

Definition 1. Let $\psi: R^{m} \rightarrow R^{n}$ be a $C^{1}$-mapping and $\psi(z)=u$. A point $z \in R^{m}$ is called a regular point of $\psi$ if $\operatorname{rank}\left(\psi^{\prime}(z)\right)=n$. A value $u \in R^{n}$ is called a regular value of $\psi$ if $z$ is a regular point of $\psi$ for all $z \in \psi^{-1}(u)$. Points and values are called singular if they are not regular. 
Lemma 1. (Inverse Image Theorem [13]). Let $\phi: U \in R^{n} \rightarrow R^{p}$ be a $C^{\alpha}$ $(\alpha>\max \{0, n-p\})$ mapping. If 0 is a regular value of $\phi$, then $\phi^{-1}(0)$ consists of some $(n-p)$-dimensional $C^{\alpha}$ manifolds.

Here, manifold means a topological space or surface and it is a subset of $R^{n}$. Applying the inverse image theorem to the homotopy method, we have the following result.

Let $H: \Omega \subset R^{n+1} \rightarrow R^{n}, H \in C^{1}$. If $\operatorname{rank}^{\prime}(x, \mu)=n$, then the connected components emanated from the equation $H(x, \mu)=0$ are $C^{1}$-smooth curves.

By $H^{\prime}(x, \mu)=\left(H_{x}^{\prime}(x, \mu), H_{\mu}^{\prime}(x, \mu)\right)$, we have if $H_{x}^{\prime}(x, \mu)$ is inverse, then $\operatorname{rank}^{\prime}(x$, $\mu)=n$. However, the condition is strong, which confines the method. To weaken the nonsingular condition and enlarge the scope of homotopy method, we introduce the well-known parameterized sard theorem which can solve this problem.

Lemma 2. (Parameterized Sard Theorem [1]). Let $V \subset R^{n}, U \subset R^{m}$ be open sets and let $\phi: V \times U \rightarrow R^{k}$ be a $C^{\alpha}$ mapping, where $\alpha>\max \{0, m-k\}$. If $0 \in R^{k}$ is a regular value of $\phi$, then for almost all $a \in V, 0$ is a regular value of $\phi_{a}=\phi(a, \cdot)$.

Parameterized sard theorem tells us that we can loose the regularity condition by introducing a suitable parameter to the homotopy equation. The full rank of jacobian matrix of the homotopy mapping $H$ reduces to the partial derivation of $H$ on the parameter having full rank.

Lemma 3. (Classification Theorem of One-Dimensional Smooth Manifolds [13]). A one-dimensional smooth manifold is homeomorphic to a unit circle or a unit interval.

We make an explanation for homeomorphic in lemma 3. A homeomorphic is also called a continuous transformation which is an equivalent relation and one to one correspondence between points in two.

From the preceding analysis, for arbitrary given $x^{(0)} \in R^{n}$ and $\mu \in(0,1]$, we construct the following homotopy equation:

$$
H\left(x, x^{(0)}, \mu\right)=(1-\mu) \Theta(x, \mu)+\mu\left(x-x^{(0)}\right)=0,
$$

where

$$
\Theta(x, \mu)=\left(\begin{array}{c}
\varphi_{C H K S}\left(x_{1}, F_{1}(x), \mu\right) \\
\vdots \\
\varphi_{C H K S}\left(x_{n}, F_{n}(x), \mu\right)
\end{array}\right)=0,
$$

and

$$
\varphi_{C H K S}\left(x_{i}, F_{i}(x), \mu\right)=\frac{1}{2}\left(x_{i}+F_{i}(x)-\sqrt{\left(x_{i}-F_{i}(x)\right)^{2}+4 \mu^{2}}\right), i=1,2, \cdots, n .
$$

It can also be rewritten by component as:

(7) $H_{i}\left(x, x^{(0)}, \mu\right)=(1-\mu) \varphi_{C H K S}\left(x_{i}, F_{i}(x), \mu\right)+\mu\left(x_{i}-x_{i}^{(0)}\right)=0, i=1,2, \cdots, n$. 
Set

$$
H_{x^{(0)}}^{-1}(0)=\left\{(x, \mu) \in R^{n} \times(0,1]: H\left(x, x^{(0)}, \mu\right)=0\right\} .
$$

Lemma 4. For almost all $x^{(0)} \in R^{n}, 0$ is a regular value of $H: R^{n} \times(0,1] \rightarrow R^{n}$, and $H_{x^{(0)}}^{-1}(0)$ consists of some smooth curves. Among them, a smooth curve $\Gamma$ starts from $\left(x^{(0)}, 1\right)$, denoted by $\Gamma_{x^{(0)}}$.

Proof. We regard the function $H$ as the variables $x, x^{(0)}, \mu$. Using $D H\left(x, x^{(0)}, \mu\right)$ to denote the Jacobian matrix of $H\left(x, x^{(0)}, \mu\right)$, we have

$$
D H\left(x, x^{(0)}, \mu\right)=\left(\frac{\partial H\left(x, x^{(0)}, \mu\right)}{\partial x}, \frac{\partial H\left(x, x^{(0)}, \mu\right)}{\partial x^{(0)}}, \frac{\partial H\left(x, x^{(0)}, \mu\right)}{\partial \mu}\right) .
$$

For all $x^{(0)} \in R^{n}$ and $\mu \in(0,1]$, recalling the homotopy equation (6), we have

$$
\frac{\partial H\left(x, x^{(0)}, \mu\right)}{\partial x^{(0)}}=-\mu I
$$

where $I$ is the identity matrix. Thus, $D H\left(x, x^{(0)}, \mu\right)$ is of full row rank. That is, 0 is a regular value of $H\left(x, x^{(0)}, \mu\right)$. By Lemma 1 and Lemma 2 , for almost all $x^{(0)} \in R^{n}, 0$ is a regular value of $H\left(x, x^{(0)}, \mu\right)$ and $H_{x^{(0)}}^{-1}(0)$ consists of some smooth curves. From $H\left(x^{(0)}, x^{(0)}, 1\right)=0$, there lies a smooth curve $\Gamma$ in $H_{x^{(0)}}^{-1}(0)$ starting from $\left(x^{(0)}, 1\right)$, denoted by $\Gamma_{x^{(0)}}$.

Lemma 5. (Boundedness of the homotopy pathway). Suppose Assumption 1 holds. When 0 is a regular value of $H\left(x, x^{(0)}, \mu\right), \Gamma_{x^{(0)}}$ is bounded on $R^{n} \times(0,1]$.

Proof. By Lemma 4, we have that 0 is a regular value of $H\left(x, x^{(0)}, \mu\right)$. If $\Gamma_{x^{(0)}} \subset$ $R^{n} \times(0,1]$ is an unbounded curve, and because $(0,1]$ is bounded, then there exists a sequence of points $\left\{\left(x^{(k)}, \mu_{k}\right)\right\} \subset \Gamma_{x^{(0)}}$ with $\left\|x^{(k)}\right\| \rightarrow \infty$. Hence, there must exist $i \in\{1, \cdots, n\}$ such that $\left|x_{i}^{(k)}\right| \rightarrow+\infty$ and $x_{i}^{(k)}$ satisfying equation (7), i.e.,

$$
\left(1-\mu_{k}\right) \varphi_{C H K S}\left(x_{i}^{(k)}, F_{i}\left(x^{(k)}\right), \mu_{k}\right)+\mu_{k}\left(x_{i}^{(k)}-x_{i}^{(0)}\right)=0 .
$$

By Remark 1, we rewrite the above equation:

$$
\left(1-\mu_{k}\right)\left(\min \left(x_{i}^{(k)}, F_{i}\left(x^{(k)}\right)\right)-\mu_{k} \alpha\left(x_{i}^{(k)}, F_{i}\left(x^{(k)}\right), \mu_{k}\right)\right)+\mu_{k}\left(x_{i}^{(k)}-x_{i}^{(0)}\right)=0,
$$

where $0<\alpha\left(x_{i}^{(k)}, F_{i}\left(x^{(k)}\right), \mu_{k}\right) \leq 1$.

Reorganizing the above equality, we obtain

$$
\begin{aligned}
& \left(1-\mu_{k}\right) \min \left(x_{i}^{(k)}, F_{i}\left(x^{(k)}\right)\right)-\mu_{k}\left(1-\mu_{k}\right) \alpha\left(x_{i}^{(k)}, F_{i}\left(x^{(k)}\right), \mu_{k}\right) \\
& \quad+\mu_{k}\left(x_{i}^{(k)}-x_{i}^{(0)}\right)=0 .
\end{aligned}
$$

For $\left|x_{i}^{(k)}\right| \rightarrow+\infty$, we have the following two possible cases: 
(1) $x_{i}^{(k)} \rightarrow-\infty$, as $k \rightarrow \infty$, there are two subcases to discuss:

(a) $F_{i}\left(x^{(k)}\right) \rightarrow-\infty$, as $k \rightarrow \infty$. In this case, for the left side of (8), the sum of the first term and the third term tends to $-\infty$, but the second term is bounded, which contradicts with the right side of (8);

(b) $F_{i}\left(x^{(k)}\right)>-\infty$, as $k \rightarrow \infty$. Then equation (8) can be simplified as follows:

$$
\begin{aligned}
\left(1-\mu_{k}\right) x_{i}^{(k)}-\mu_{k}\left(1-\mu_{k}\right) \alpha\left(x_{i}^{(k)}, F_{i}\left(x^{(k)}\right), \mu_{k}\right)+\mu_{k}\left(x_{i}^{(k)}-x_{i}^{(0)}\right) & =0 \\
\Longleftrightarrow x_{i}^{(k)}-\mu_{k}\left(1-\mu_{k}\right) \alpha\left(x_{i}^{(k)}, F_{i}\left(x^{(k)}\right), \mu_{k}\right)-\mu_{k} x_{i}^{(0)} & =0
\end{aligned}
$$

Since $\mu_{k}, \alpha\left(x_{i}^{(k)}, F_{i}\left(x^{(k)}\right), \mu_{k}\right)$ and $x_{i}^{(0)}$ are all bounded, so (9) is a contradiction. Hence, this case can not occur.

(2) $x_{i}^{(k)} \rightarrow+\infty$, as $k \rightarrow \infty$, there are also two subcases to discuss:

(c) $F_{i}\left(x^{(k)}\right) \rightarrow+\infty$, as $k \rightarrow \infty$. The proof is similar to case (a). It is impossible to occur.

(d) $F_{i}\left(x^{(k)}\right)<+\infty$, as $k \rightarrow \infty$. Then equation (8) can be reorganized as:

$$
\left(1-\mu_{k}\right) F_{i}\left(x^{(k)}\right)-\mu_{k}\left(1-\mu_{k}\right) \alpha\left(x_{i}^{(k)}, F_{i}\left(x^{(k)}\right), \mu_{k}\right)+\mu_{k}\left(x_{i}^{(k)}-x_{i}^{(0)}\right)=0,
$$

so we have

$$
F_{i}\left(x^{(k)}\right)=-\frac{\mu_{k}}{1-\mu_{k}}\left(x_{i}^{(k)}-\left(1-\mu_{k}\right) \alpha\left(x_{i}^{(k)}, F_{i}\left(x^{(k)}\right), \mu_{k}\right)-x_{i}^{(0)}\right) .
$$

Since $\mu_{k} \in(0,1), \alpha\left(x_{i}^{(k)}, F_{i}\left(x^{(k)}\right), \mu_{k}\right)$ and $x_{i}^{(0)}$ are all bounded when $k$ is sufficiently large, so the right side of equation (10) is non-positive, which contradicts with Assumption 1.

Combing (1) and (2) together, we have that $\Gamma_{x^{(0)}}$ is bounded on $R^{n} \times(0,1]$.

Lemma 6. For $x^{(0)} \in R^{n}, H\left(x, x^{(0)}, 1\right)=0$ has a unique solution $\left(x^{(0)}, 1\right)$.

Proof. When $\mu=1$, by homotopy equation (6), it is easy to obtain the conclusion.

Theorem 1. (Convergence of the homotopy pathway). Let $F$ be twice continuously differentiable. Suppose Assumption 1 holds. Then, for almost all $x^{(0)} \in R^{n}$, homotopy equation (6) determines a bounded smooth curve $\Gamma_{x^{(0)}} \subset R^{n} \times(0,1]$ which starts from $\left(x^{(0)}, 1\right)$ and approaches the hyperplane at $\mu=0$. When $\mu \rightarrow 0$, the limit set $T \times\{0\} \subset R^{n} \times\{0\}$ of $\Gamma_{x^{(0)}}$ is nonempty, and the $x$-component $x^{*}$ of every point $\left(x^{*}, 0\right)$ in $T \times\{0\}$ solves $(1)$. 
Proof. By Lemma 4, for almost all $x^{(0)} \in R^{n}, 0$ is a regular value of $H$ and $H_{x^{(0)}}^{-1}(0)$ contains a smooth curve $\Gamma_{x^{(0)}} \subset R^{n} \times(0,1]$ starting from $\left(x^{(0)}, 1\right)$. By Lemma $3, \Gamma_{x(0)} \subset R^{n} \times(0,1]$ must be homeomorphic to a unit circle or the unit interval $(0,1]$ or $[0,1]$, where 1 is regarded as the image of the starting point. In other words, the curve $\Gamma_{x^{(0)}}$ will return to $\left(x^{(0)}, 1\right)$, terminates in or approaches to the boundary of $R^{n} \times[0,1]$ or goes to infinity.

Notice that $\left.\frac{\partial H\left(x, x^{(0)}, \mu\right)}{\partial x}\right|_{x=x^{(0)}, \mu=1}=I$ is nonsingular, as a result, $\left(x^{(0)}, 1\right)$ is not a multiple solution of $H\left(x, x^{(0)}, \mu\right)=0$. Hence, $\Gamma_{x^{(0)}}$ is not homeomorphic to a unit circle and it is homeomorphic to the unit interval. The limit points of $\Gamma_{x^{(0)}}$ must lie in $R^{n} \times[0,1]$. Let $\left(x^{*}, \mu^{*}\right)$ be a limit point of $\Gamma_{x^{(0)}}$. Only the following two cases are possible:

(i) $\left(x^{*}, \mu^{*}\right) \in R^{n} \times\{1\}$;

(ii) $\left(x^{*}, \mu^{*}\right) \in R^{n} \times\{0\}$.

Because equation $H\left(x, x^{(0)}, 1\right)=0$ has only one solution $\left(x^{(0)}, 1\right)$ in $R^{n} \times\{1\}$, Case (i) is impossible. Therefore, Case (ii) is the only possible case. If $\left(x^{*}, 0\right)$ is the limit point of $\Gamma_{x^{(0)}}$, by Lemma 5 , we have $\left(x^{*}, 0\right)$ is a solution of (6) and $x^{*}$ is the solution of the NCP. The proof is completed.

By Theorem 1, for almost all $x^{(0)} \in R^{n}$, if Assumption 1 holds, then the homotopy equation (6) generates a smooth curve $\Gamma_{x^{(0)}}$, which is called as the homotopy pathway. Tracing numerically $\Gamma_{x^{(0)}}$ from $\left(x^{(0)}, 1\right)$ until $\mu \rightarrow 0$, one can find a solution of (1). Letting $s$ be the arc-length of $\Gamma_{x(0)}$, we can parameterize $\Gamma_{x^{(0)}}$ with respect to $s$. That is, there exist continuously differentiable functions $x(s), \mu(s)$ such that

$$
\begin{aligned}
H(x(s), \mu(s)) & =0, \\
\|\dot{x}(s), \dot{\mu}(s)\| & =1, \\
x(0)=x^{(0)}, \mu(0) & =1 .
\end{aligned}
$$

Differentiating the first equality of (11), we obtain the following result.

Theorem 2. The homotopy path $\Gamma_{x^{(0)}}$ is determined by the following initial value problem to the system of ordinary differential equations

$$
\begin{aligned}
D H_{x(0)}(x(s), \mu(s))\left(\begin{array}{c}
\dot{x} \\
\dot{\mu}
\end{array}\right) & =0, \\
\|\dot{x}(s), \dot{\mu}(s)\| & =1, \\
x(0)=x^{(0)}, \mu(0) & =1 .
\end{aligned}
$$

And the $x$-component of the solution point $\left(x\left(s^{*}\right), \mu\left(s^{*}\right)\right)$ of $(11)$, for $\mu\left(s^{*}\right)=0$, is the solution of $N C P(1)$. 
Proof. Differentiating the first equality of (11) on $s$, we have

$$
H_{x}^{\prime}(x, \mu) \frac{d x}{d s}+H_{\mu}^{\prime}(x, \mu) \frac{d \mu}{d s}=0 .
$$

If $s$ is the arc-length of $\Gamma_{x^{(0)}}$, then there holds

$$
\left\|y^{\prime}\right\| \equiv\left\|\left(\begin{array}{c}
\frac{d x}{d s} \\
\frac{d \mu}{d s}
\end{array}\right)\right\|=1,
$$

where $y=(x, \mu)^{T} \in R^{n+1},\|y\|=\|y\|_{2}$. Therefore, we obtain the following system of ordinary differential equations

$$
\left\{\begin{array}{l}
\left(H_{x}^{\prime}(x, \mu), H_{\mu}^{\prime}(x, \mu)\right) u=0 \\
(u, u)=1 \\
\frac{d y}{d s}=u \\
y(0)=\left(\begin{array}{l}
x(0) \\
\mu(0)
\end{array}\right)=\left(\begin{array}{c}
x^{(0)} \\
1
\end{array}\right)
\end{array}\right.
$$

Note that

$$
D H\left(x, x^{(0)}, \mu\right)=\left(H_{x}^{\prime}\left(x, x^{(0)}, \mu\right), H_{x^{(0)}}^{\prime}\left(x, x^{(0)}, \mu\right), H_{\mu}^{\prime}\left(x, x^{(0)}, \mu\right)\right)
$$

and $\operatorname{rank}\left(H_{x^{(0)}}^{\prime}\left(x, x^{(0)}, \mu\right)\right)=n$, by parameterized sard theorem, we have for almost all $x^{(0)} \in R^{n}, \operatorname{rank}\left(D H\left(x, x^{(0)}, \mu\right)\right)=n$. It is easy to prove the matrix

$$
\left(\begin{array}{c}
H_{x}^{\prime}(x, \mu), H_{\mu}^{\prime}(x, \mu) \\
u^{T}
\end{array}\right)
$$

is nonsingular. Hence, the system consisting of the first and the second equations in (12) is solvable on $u$. Solving the following Cauchy problem, we can derive the curve $\Gamma_{x^{(0)}}$.

$$
\left\{\begin{array}{l}
\frac{d y}{d s}=u \\
y(0)=\left(\begin{array}{l}
x(0) \\
\mu(0)
\end{array}\right)=\left(\begin{array}{c}
x^{(0)} \\
1
\end{array}\right)
\end{array}\right.
$$

The proof is completed. 


\section{NuMERICAL EXPERIMENTS}

In this section, we formulate a predictor-corrector procedure (c.f., e.g., $[1,17])$ to numerically trace the homotopy path which is generated by the homotopy equation $H(w, \mu)=0$ in the subset $\Omega=\{(w, \mu): G(w) \leq 0, \mu \in(0,1]\}$, where $G: R^{p} \rightarrow R^{q}$ and $H: R^{p} \times[0,1] \rightarrow R^{p}$, starting from $\left(w^{(0)}, 1\right)$ with $w^{(0)} \in \Omega^{0}=\{w: G(w)<0\}$.

Algorithm 3.1. (Euler-Newton method).

Step 0. Input $w^{(0)}$, choose an initial step length $h_{0}>0$ and three small positive numbers $\epsilon_{1}, \epsilon_{2}, \epsilon_{3}$ with $\epsilon_{1}<\epsilon_{2}$ and set $\mu_{0}=1, k:=0$.

Step 1. Determine the predictor direction $\eta^{(k)}$ :

(a) Compute a unit tangent vector $\xi^{(k)}$ such that $D H\left(w^{(k)}, \mu_{k}\right) \xi^{(k)}=0$;

(b) Determine the predictor direction $\eta^{(k)}$ :

When $k=0$, if the last element of $\xi^{(0)}$ is negative, then $\eta^{(0)}=\xi^{(0)}$, else $\eta^{(0)}=-\xi^{(0)}$. Computing $\sigma=\operatorname{sign} \operatorname{det}\left(\begin{array}{c}D H\left(w^{(0)}, 1\right) \\ \eta^{(0)^{T}}\end{array}\right)$;

When $k \geq 1$, if sign $\operatorname{det}\left(\begin{array}{c}D H\left(w^{(k)}, \mu_{k}\right) \\ \xi^{(k)^{T}}\end{array}\right)=\sigma$, then $\eta^{(k)}=\xi^{(k)}$, else $\eta^{(k)}=$ $-\xi^{(k)}$

Step 2. Compute the predictor point $\left(\bar{w}^{(k)}, \bar{\mu}_{k}\right)=\left(w^{(k)}, \mu_{k}\right)+\alpha^{l} h_{k} \eta^{(k)}$, where $l$ is the least positive integer such that $\left(\bar{w}^{(k)}, \bar{\mu}_{k}\right) \in \Omega^{0} \times(0,1]$.

Step 3. Compute a corrector point $\left(w^{(k+1)}, \mu_{k+1}\right)$ :

$$
\left(w^{(k+1)}, \mu_{k+1}\right)=\left(\bar{w}^{(k)}, \bar{\mu}_{k}\right)-\left(D H\left(\bar{w}^{(k)}, \bar{\mu}_{k}\right)\right)^{\dagger} H\left(\bar{w}^{(k)}, \bar{\mu}_{k}\right) .
$$

If $\left(w^{(k+1)}, \mu_{k+1}\right) \notin \Omega^{0} \times(0,1]$, then $h_{k}=0.75 h_{k}$, go to Step 2, else continue.

If $\left\|H\left(w^{(k+1)}, \mu_{k+1}\right)\right\| \leq \epsilon_{1}, h_{k+1}=\min \left\{h_{0}, 2 h_{k}\right\}$, go to Step 3;

If $\left\|H\left(w^{(k+1)}, \mu_{k+1}\right)\right\| \in\left(\epsilon_{1}, \epsilon_{2}\right), h_{k+1}=h_{k}$, go to Step 3;

If $\left\|H\left(w^{(k+1)}, \mu_{k+1}\right)\right\| \geq \epsilon_{2}, h_{k+1}=h_{k} / 2, k:=k+1$, go to Step 2 .

Step 4. If $\mu_{k+1} \leq \epsilon_{3}$, then stop, else $k:=k+1$, and go to Step 1. In the Step 3 of Algorithm,

$$
(D H(w, \mu))^{\dagger}=D H(w, \mu)^{T}\left(D H(w, \mu) D H(w, \mu)^{T}\right)^{-1}
$$

is the Moore-Penrose inverse of $D H(w, \mu)$.

We use the above path following algorithm to implement the smoothing homotopy method with the homotopy equation (6) and the homotopy method in [16] for solving the NCP in MATLAB. For the former, let $w=x$ and take an interior starting point 
and an exterior starting point to test, respectively. For the latter, the homotopy equation refers to (3) and $G(w)=-w$ and we take the same interior point to compare the two methods in iteration time and iteration steps. For all test problems, we take the accuracy parameters $\epsilon_{1}=10^{-4}, \epsilon_{2}=10^{-3}, \epsilon_{3}=10^{-6}, \mu_{0}=1.0$. And the numerical results are listed in the tables, where two initial points $x^{(0)}$ are an interior point and an exterior point, respectively, A1 denotes our smoothing homotopy method and $\mathbf{A 2}$ denotes homotopy method in [16], IT denotes the number of iterations, ACPU denotes the average total cost time in second for solving the problem among the ten runs, $x^{*}$ denotes the approximate solution of the considered problem, $F\left(x^{*}\right)$ denotes the value of the function $F$ at $x^{*}$, and $\mu^{*}$ denotes the value when the algorithm terminates. Numerical tests indicate that our smoothing homotopy method for solving the NCP is more effective.

Example 3.1. ([8], Example 3).

$$
F\left(x_{1}, x_{2}, x_{3}, x_{4}\right)=\left(\begin{array}{c}
3 x_{1}^{2}+2 x_{1} x_{2}+2 x_{2}^{2}+x_{3}+3 x_{4}-6 \\
2 x_{1}^{2}+x_{1}+x_{2}^{2}+10 x_{3}+2 x_{4}-2 \\
3 x_{1}^{2}+x_{1} x_{2}+2 x_{2}^{2}+2 x_{3}+9 x_{4}-9 \\
x_{1}^{2}+3 x_{2}^{2}+2 x_{3}+3 x_{4}-3
\end{array}\right)
$$

In this example, we take two different initial points to test. The experiment results see Table 1.

Table 1: The numerical results of Example 3.1

\begin{tabular}{|c|c|c|c|c|c|c|}
\hline$x_{0}$ & method & ACPU & IT & $x^{*}$ & $F\left(x^{*}\right)$ & $\mu^{*}$ \\
\hline$(1,1,1,1)^{T}$ & A1 & 0.0109 & 18 & $(1,0,3,0)^{T}$ & $(0,31,0,4)^{T}$ & $2.9401 \times 10^{-7}$ \\
& A2 & 0.2298 & 348 & $(1,0,3,0)^{T}$ & $(0,31,0,4)^{T}$ & $4.5323 \times 10^{-7}$ \\
\hline$(-1,0,0,-0.5)^{T}$ & A1 & 0.0126 & 19 & $(1,0,3,0)^{T}$ & $(0,31,0,4)^{T}$ & $3.3511 \times 10^{-7}$ \\
\hline
\end{tabular}

Example 3.2. ([10]).

$$
F(x)=\left(\begin{array}{c}
3 x_{1}^{2}+2 x_{1} x_{2}+2 x_{2}^{2}+x_{3}+3 x_{4}-6 \\
2 x_{1}^{2}+x_{2}^{2}+x_{1}+3 x_{3}+2 x_{4}-2 \\
3 x_{1}^{2}+x_{1} x_{2}+2 x_{2}^{2}+2 x_{3}+3 x_{4}-1 \\
x_{1}^{2}+3 x_{2}^{2}+2 x_{3}+3 x_{4}-3
\end{array}\right)
$$

In this example, we take two different initial points and the experiment results see Table 2.

Table 2: The numerical results of Example 3.2

\begin{tabular}{|c|c|c|c|c|c|}
\hline$x_{0}$ & method & ACPU & IT & $x^{*}$ & $\mu^{*}$ \\
\hline$(1,1,1,1)^{T}$ & A1 & 0.0110 & 17 & $(1.2247,0.0000,0.0000,0.5000)^{T}$ & $1.2920 \times 10^{-7}$ \\
& A2 & 0.0701 & 104 & $(1.2247,0.0000,0.0000,0.5000)^{T}$ & $4.4071 \times 10^{-7}$ \\
\hline$(-1,-1,1,1)^{T}$ & A1 & 0.0157 & 24 & $(1.2247,0.0000,0.0000,0.5000)^{T}$ & $6.9288 \times 10^{-7}$ \\
\hline
\end{tabular}


Example 3.3. ([15], Example 4). Consider the convex prgramming problem

$$
\min \theta(x)=\exp \left(\sum_{i=1}^{5}\left(x_{i}-i+2\right)^{2}\right) \quad \text { subject to } x \geq 0 .
$$

The Kuhn-Tucker optimality conditions are applied to this problem, which results in a complementarity problem with

$$
F(x)=\nabla \theta(x)=2 \exp \left(\Sigma_{i=1}^{5}\left(x_{i}-i+2\right)^{2}\right)\left(\begin{array}{c}
x_{1}+1 \\
x_{2} \\
x_{3}-1 \\
x_{4}-2 \\
x_{5}-3
\end{array}\right)
$$

In this example, we take two different initial points and the experiment results see Table 3.

Table 3: The numerical results of Example 3.3

\begin{tabular}{|c|c|c|c|c|c|c|}
\hline$x_{0}$ & method & ACPU & IT & $x^{*}$ & $F\left(x^{*}\right)$ & $\mu^{*}$ \\
\hline$(1,1,2,3,4)^{T}$ & A1 & 0.0127 & 28 & $(0,0,1,2,3)^{T}$ & $(5.4366,0,0,0,0)^{T}$ & $7.2493 \times 10^{-7}$ \\
& A2 & fails & - & - & - & - \\
\hline$(-1,2,2,3,4)^{T}$ & A1 & 0.0110 & 22 & $(0,0,1,2,3)^{T}$ & $(5.4366,0,0,0,0)^{T}$ & $3.8575 \times 10^{-7}$ \\
\hline
\end{tabular}

Example 3.4. ([9]). This example is a modification of Mathiesen [12]

$$
\begin{aligned}
& F_{1}(x)=-x_{2}+x_{3}+x_{4}, \\
& F_{2}(x)=x_{1}-\left(4.5 x_{3}+2.7 x_{4}\right) /\left(x_{2}+1\right), \\
& F_{3}(x)=5-x_{1}-\left(0.5 x_{3}+0.3 x_{4}\right) /\left(x_{3}+1\right), \\
& F_{4}(x)=3-x_{1} .
\end{aligned}
$$

This example has infinitely many solutions $(\lambda, 0,0,0)$, where $\lambda \in[0,3]$. The test results for Example 3.4 are listed in Table 4 for different starting points.

Table 4: The numerical results of Example 3.4

\begin{tabular}{|c|c|c|c|c|c|c|}
\hline$x_{0}$ & method & ACPU & IT & $x^{*}$ & $F\left(x^{*}\right)$ & $\mu^{*}$ \\
\hline$(2,2,2,2)^{T}$ & A1 & 0.0205 & 39 & $(0.0013,0.0000,0.0000,0.0000)^{T}$ & $(0.0000,0.0012,4.9987,2.9987)^{T}$ & $8.5437 \times 10^{-7}$ \\
& A2 & 0.1719 & 154 & $(1.4174,0.0000,0.0000,0.0000)^{T}$ & $(0.0000,1.4174,3.5826,1.5826)^{T}$ & $6.9454 \times 10^{-7}$ \\
\hline$(-1,1,1,-1)^{T}$ & A1 & 0.0172 & 29 & $(0.0010,0.0000,0.0000,0.0000)^{T}$ & $(0.0000,0.0010,4.9990,2.9990)^{T}$ & $5.1620 \times 10^{-7}$ \\
\hline
\end{tabular}

\section{ACKNOWLEDGMENTS}

The authors like to express our thanks to anonymous reviewers, whose precise remarks and insightful observations lead to an improved version of the paper. 


\section{REFERENCES}

1. E. L. Allgower and K. Georg, Numerical Continuation Methods: An Introduction, Springer-Vergal, Berlin, New York, 1990.

2. B. T. Chen and P. T. Harker, Smooth approximations to nonlinear complementarity problems, SIAM Journal on Optimization, 7(2) (1997), 403-420.

3. B. L. Chen and C. F. Ma, A new smoothing Broyden-like method for solving nonlinear complementarity problem with a $P_{0}$ function, J. Glob. Optim., 51 (2011), 473-495.

4. M. C. Ferris and J.-S. Pang, Engineering and economic applications of complementarity problems, SIAM Review, 39 (1997), 669-713.

5. M. Fukushima, Merit functions for variational inequality and complementarity problems, in Nonlinear Optimization and Applications, G. Di Pillo and F. Giannessi eds., Plenum Publishing Corporation, New York, pp. 155-170, 1996.

6. P. T. Harker and J.-S. Pang, Finite-dimensional variational inequality and nonlinear complementarity problem: a survey of theory, algorithms and applications, Mathematical Progamming, 48 (1990), 161-220.

7. P. T. Harker, Complementarity problem, in Handbook of Global Optimization, R. Horst and P. Pardalos, eds., Kluwer Academic Publishers, Boston, pp. 271-338, 1995.

8. H. Jiang and L. Qi, A new nonsmooth equations approach to nonliner complementarity problems, SIAM Journal on Control and Optimization, 35 (1997), 178-193.

9. C. Kanzow, Some equation-based methods for the nonlinear complementarity problem, Optimization Methods and Software, 3 (1994), 327-340.

10. M. Kojima and S. Shindo, Extensions of Newton and quasi-Newton methods to systems of $P C^{1}$ equations, Journal of Operation Research Society of Japan, 29 (1986), 352-374.

11. T. De Luca, F. Facchinei and C. Kanzow, A semismooth equation approach to the solution of nonlinear complementarity problems, Mathematical Programming, 75(3) (1996), 407 439.

12. L. Mathiesen, An algorithm based on a sequence of linear complementarity problems applied to a Walrasian equilibrium model: an example, Mathematical Programming, 37 (1987), 1-18.

13. G. L. Naber, Topological Method in Euclidean Space, Cambridge Univ. Press, London, 1980.

14. D. Sun, A regularization Newton method for solving nonlinear complementarity problems, Applied Mathematics and Optimization, 40 (1999), 315-339.

15. L. T. Watson, Solving the nonlinear complementarity problem by a homotopy method, SIAM Journal on Control and Optimization, 17(1) (1979), 36-46.

16. Q. Xu and C. Dang, A new homotopy method for solving non-linear complementarity problems, Optimization, 57(5) (2008), 681-689. 
17. B. Yu and Z. Lin, Homotopy method for a class of nonconvex Brouwer fixed point problems, Applied Mathematics and Computation, 74 (1996), 65-77.

Xiaona Fan, Tingting Xu and Furong Gao

College of Science

Nanjing University of Posts and Telecommunications

Nanjing, Jiangsu 210023

P. R. China

E-mail: xiaonafan@126.com 